\title{
Méthodes simples de modélisation mathématique pour évaluer le risque de transmission du SRAS-CoV-2 pendant les rassemblements
}

\author{
David Champredon ${ }^{1 \star}$, Aamir Fazil ${ }^{1}$, Nicholas H Ogden²
}

\section{Résumé}

Contexte : Les rassemblements peuvent contribuer de manière significative à la propagation du coronavirus du syndrome respiratoire aigu sévère 2 (SRAS-CoV-2). C'est pourquoi les interventions de santé publique ont cherché à limiter les rassemblements non répétés ou récurrents pour enrayer la pandémie de maladie à coronavirus 2019 (COVID-19). Malheureusement, l'éventail des différents types de rassemblements ne permet pas de donner des directives précises pour fixer des paramètres limitatifs (e.g. la taille totale du rassemblement, le nombre de cohortes, le degré de distanciation physique).

Méthodes : Nous avons utilisé un cadre de modélisation générique basé sur les principes fondamentaux de probabilité pour dériver des formules simples permettant d'évaluer les risques d'introduction et de transmission liés aux rassemblements ainsi que l'efficacité potentielle de certaines stratégies de dépistage pour atténuer ces risques.

Résultats : Le risque d'introduction peut être évalué de manière générale en fonction de la prévalence de la population et de la taille du rassemblement, tandis que le risque de transmission au cours d'un rassemblement dépend principalement de la taille du rassemblement. Pour les rassemblements récurrents, la structure de la cohorte n'a pas d'effet significatif sur la transmission entre cohortes. Les stratégies de test peuvent atténuer les risques, mais la fréquence et la performance des tests permettent d'établir un équilibre entre la détection et les faux positifs.

Conclusion : La généralité du cadre de modélisation utilisé ici permet de démêler les différents facteurs des risques de transmission au cours des rassemblements. Ce cadre pourra éclairer la prise de décisions en matière de santé publique.
Cette oeuvre est mise à la disposition selon les termes de la licence internationale Creative Commons Attribution 4.0

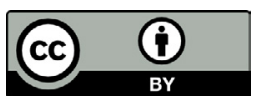

Affiliations

1 Division des sciences des risques pour la santé publique, Laboratoire national de microbiologie, Agence de la santé publique du Canada, Guelph, ON

${ }^{2}$ Division des sciences des risques pour la santé publique, Laboratoire national de microbiologie, Agence de la santé publique du Canada, St Hyacinthe, QC et Guelph, ON

*Correspondance : david.champredon@canada.ca

Citation proposée : Champredon D, Fazil A, Ogden NH. Méthodes simples de modélisation mathématique pour évaluer le risque de transmission du SRAS-CoV-2 pendant les rassemblements. Relevé des maladies transmissibles au Canada 2021;47(4):201-12. https://doi.org/10.14745/ccdr.v47i04a02f

Mots-clés : risques de transmission, SRAS-CoV-2, COVID-19, Canada, rassemblements, modélisation mathématique

\section{Introduction}

Depuis l'émergence du coronavirus du syndrome respiratoire aigu sévère 2 (SRAS-CoV 2) à la fin de 2019, on dispose de données qui confirment que les rassemblements peuvent augmenter les risques de transmission du SRAS-CoV 2 dans la population et qu'ils peuvent même causer une super propagation (1-3). Pour ralentir la progression de l'épidémie de maladie à coronavirus 2019 (COVID-19), les décideurs ont limité, entre autres choses, le nombre de personnes désirant se réunir pour des raisons tant personnelles que professionnelles. À première vue, la taille des rassemblements est directement liée au taux d'infection; par conséquent, en la limitant, on espère minimiser la transmission de la COVID-19.

Au-delà de ce simple constat, il est difficile d'évaluer l'efficacité des restrictions sur les rassemblements. Les rassemblements peuvent prendre une multitude de formes différentes, de la 
fête d'anniversaire d'un enfant à l'intérieur avec des invités locaux à des mariages et des congrès dont les participants viennent de communautés diverses. Ces différentes formes reflètent la diversité des valeurs des variables qui déterminent la transmission de la maladie pendant le rassemblement (e.g. les associations de participants, les taux et les modes de contact, la durée du rassemblement, la prévalence chez les participants au début de l'événement).

Des modèles de transmission détaillés, adaptés à des événements spécifiques, ont servi à évaluer la complexité des risques de transmission et à fournir des indications sur le rôle qu'y jouent les rassemblements. Une analyse approfondie des études de modélisation évaluant les risques liés aux rassemblements a démontré que tous les modèles prouvent qu'en limitant la taille des rassemblements, on limite la transmission du SRAS-CoV-2 (3). Malheureusement, nous disposons rarement de données suffisantes pour paramétrer de tels modèles de transmission "sur mesure ". D'ailleurs même si nous en disposions, il serait difficile d'en généraliser les résultats.

Nous tentons ici d'évaluer les risques de transmission du SRAS-CoV-2 au cours des rassemblements (qu'ils soient exceptionnels ou fréquents) en utilisant des cadres de modélisation relativement simples et génériques. Nous nous concentrons sur les risques qui se posent généralement pendant des rassemblements, sur les risques d'introduction et de transmission pendant les rassemblements ainsi que sur deux méthodes couramment utilisées pour atténuer les risques : les tests de dépistage sur les participants et (pour les rassemblements répétés) sur toute la cohorte. Bien que les résultats présentés ici ne fournissent pas vraiment d'orientations précises pour des rassemblements particuliers, ils s'appliquent néanmoins à divers degrés, à différents types de rassemblements et ils éclairent la prise de décisions à haut niveau en matière de santé publique. Au fur et à mesure que l'on obtient des données quantitatives plus détaillées sur les aspects des risques que posent les rassemblements (comme la ventilation, la densité des groupes de participants, les niveaux de vocalisation) (3), on peut affiner les paramètres de ce type de modélisation afin d'estimer avec plus de précision les risques que posent les différents rassemblements.

\section{Rassemblements uniques}

Les rassemblements uniques ne se produisent qu'une fois, ou se produisent à des intervalles qui permettent de les considérer comme étant uniques. II peut s'agir, par exemple, de funérailles, de mariages ou de conférences.

\section{Risques d'introduction}

Le premier déterminant du risque des rassemblements est la probabilité qu'au moins une personne contagieuse y participe. Une approche générale consisterait à supposer que les personnes contagieuses sont choisies au hasard dans une population qui se mélange de manière homogène (une hypothèse prudente lorsqu'on considère le risque de transmission). Avec ces hypothèses, le risque d'avoir une personne contagieuse dans un rassemblement est proportionnel à la prévalence dans la population générale (appelée ici prév). La probabilité qu'au moins une personne contagieuse participe à un rassemblement de taille $N$ est la suivante

$$
p_{\text {intro }}=1-(1-\text { prév })^{N}
$$

Cette simple équation donne différentes valeurs pour un décideur. La variable $p_{\text {intro }}$ est la probabilité qu'au moins une personne contagieuse participe à un rassemblement de taille $N$ dans un contexte où la prévalence de la population est prév. Un simple réajustement de l'équation fournit la plus grande taille possible du rassemblement pour un niveau acceptable prédéterminé de risque d'introduction pour une prévalence d'infection donnée dans la population participant au rassemblement :

$$
N=\log \left(1-p_{\text {intro }}\right) / \log (1-\text { prév })
$$

Un autre ajustement fournit le niveau de prévalence dans la population qui dépasserait le niveau acceptable prédéterminé de risque d'introduction pour un rassemblement d'une taille donnée :

$$
\text { prév }=1-\left(1-p_{\text {intro }}\right)^{1 / N}
$$

Soulignons que si ces trois simples équations ne peuvent prétendre à la précision d'un rassemblement spécifique, elles peuvent aider à comprendre comment ces trois variables sont reliées. Les relations entre la taille du rassemblement, la prévalence dans la collectivité et la tolérance au risque d'introduction $\left(p_{\text {intro }}\right)$ sont illustrées dans la figure 1.

L'hypothèse selon laquelle la prévalence dans la population source est la même que celle de la sous-population participant au rassemblement est commode, mais peut ne pas être réaliste pour les rassemblements qui attirent des personnes venant de sous-populations plus ou moins susceptibles d'être infectées.

Une façon simple de représenter cette hétérogénéité est de modifier directement la prévalence en fonction de la surexposition ou de la sous-exposition supposée des participants au rassemblement. La prévalence ajustée pour le groupe prev $v_{G}$ peut être simplement calculée à partir de la prévalence de base. Si nous connaissons le risque relatif du groupe par rapport à l'ensemble de la population, et si nous connaissons le rapport des cotes de contagion pour ce groupe, nous avons:

$$
\text { prév }_{G}=R R \times \text { prév, or prév }{ }_{G}=\left(1+\frac{1-p r e ́ v}{\text { OR×prév }}\right)^{-1}
$$

Par exemple, si 1) la prévalence actuelle des contagions par le SRAS-CoV 2 dans la population qui participe au rassemblement est prév $=0,5 \%, 2$ ) les caractéristiques démographiques du rassemblement sont similaires à celles de l'ensemble de la population et 3 ) nous décidons que la probabilité maximale acceptable qu'une personne contagieuse se joigne à ce rassemblement est $p_{\text {intro }}=20 \%$, alors le nombre de participants 
Figure 1 : Relations entre la taille du rassemblement, la prévalence dans la population et la tolérance au risque d'introduction

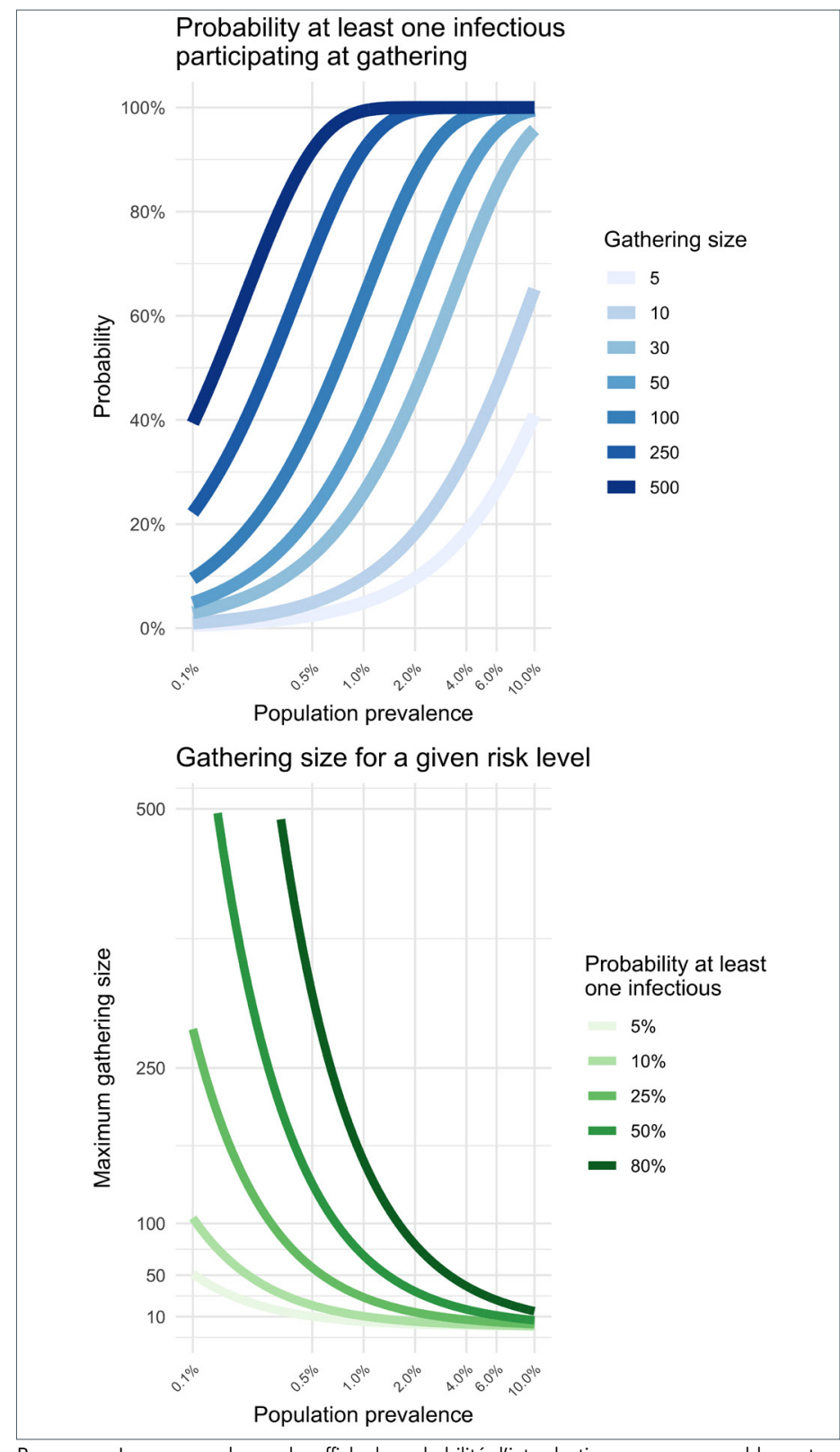

Remarque : Le panneau de gauche affiche la probabilité d'introduction pour un rassemblement d'une taille et d'une prévalence données. Le panneau de droite montre la taille maximale de rassemblement pour une prévalence et un risque d'introduction donnés (Figure en anglais seulement)

au rassemblement ne devrait pas dépasser $N=44$. Toutefois, si I'on considère un rassemblement où un groupe de participants risque cinq fois plus d'être infecté que la population générale (prév $v_{G}=5 \times$ prév $=2,5 \%$ ), le nombre de participants à ce rassemblement ne devrait pas être supérieur à neuf.

\section{Risque de transmission pendant le rassemblement}

Une fois que l'on a calculé la probabilité qu'une personne infectée participe au rassemblement, il faut se poser la question suivante : "Quel est le risque que cette personne transmette l'agent pathogène à d'autres participants susceptibles?".

Si nous supposons que les contacts entre participants est homogène pendant un rassemblement de $N$ personnes auquel participent $I$ personnes contagieuses et que toutes les personnes susceptibles entreront en contact avec $C$ personnes différentes (contagieuses ou non) pendant le rassemblement, le nombre minimum de transmissions à prévoir pendant ce rassemblement est le suivant :

$$
n_{\text {transm }}=(N-I) \times\left(1-\left(1-\frac{I}{N-1} p_{t r}\right)^{C}\right)
$$

où $C$ est le nombre de contacts pendant le rassemblement avec une personne contagieuse, et $p_{t r}$ est la probabilité de transmissions dues à un contact avec des personnes contagieuses (voir l'annexe pour plus de détails). Les variables $C$ et $p_{t r}$ sont spécifiques au contexte et doivent être calibrées en fonction des meilleures données disponibles à partir des analyses épidémiologiques et des études de recherche. II peut être utile de travailler avec une gamme d'estimations qui produiront des limites supérieures et inférieures pour $n_{\text {transm }}$. La formule ci dessus est assez simple pour être calculée dans un tableur et peut aider à prévoir le rôle de la taille du rassemblement et des mesures qui contribuent à réduire la probabilité de transmission (e.g. le port du masque) ou le nombre de contacts (e.g. la distanciation physique).

La figure 2 montre $n_{\text {transm }}$ pour différentes valeurs de la taille du rassemblement et de participants contagieux. Par exemple, on peut s'attendre à ce qu'il y ait environ quatre transmissions au cours d'un rassemblement de 10 personnes où se trouvent deux participants contagieux (figure 2, panneau central), à ce que le taux de contacts s'élève en moyenne à 30 par personne et à ce que la probabilité de transmission soit $p_{t r}=10 \%$. Lorsqu'une seule personne contagieuse est présente à un rassemblement (panneau de gauche), le nombre attendu de transmissions est approximativement le même pour différentes tailles de rassemblement. Ceci est principalement dû au fait que la probabilité qu'une personne susceptible rencontre une personne contagieuse est faible. Le résultat est très différent avec la présence de cinq personnes contagieuses (figure 2). Dans ce cas, la probabilité que les personnes susceptibles rencontrent des personnes contagieuses dans la foule augmente et, par conséquent, le nombre de transmissions qui pourraient se produire augmente également.

Pour les très grands rassemblements, nous pouvons raisonnablement supposer que le nombre de participants contagieux devrait être approximativement égal à la prévalence de la population, en supposant que le rassemblement soit un échantillon aléatoire de la population.

Si $C_{\max }$ est le nombre maximum de contacts qu'une personne contagieuse peut faire pendant le rassemblement, alors $A=S /\left(C_{\max } p_{t r}\right)$ est le nombre minimum de personnes contagieuses nécessaires pour avoir une chance $d^{\prime}$ 'infecter 
Figure 2 : Effet de la taille du rassemblement et du nombre de personnes contagieuses sur le nombre minimum de transmissions secondaires

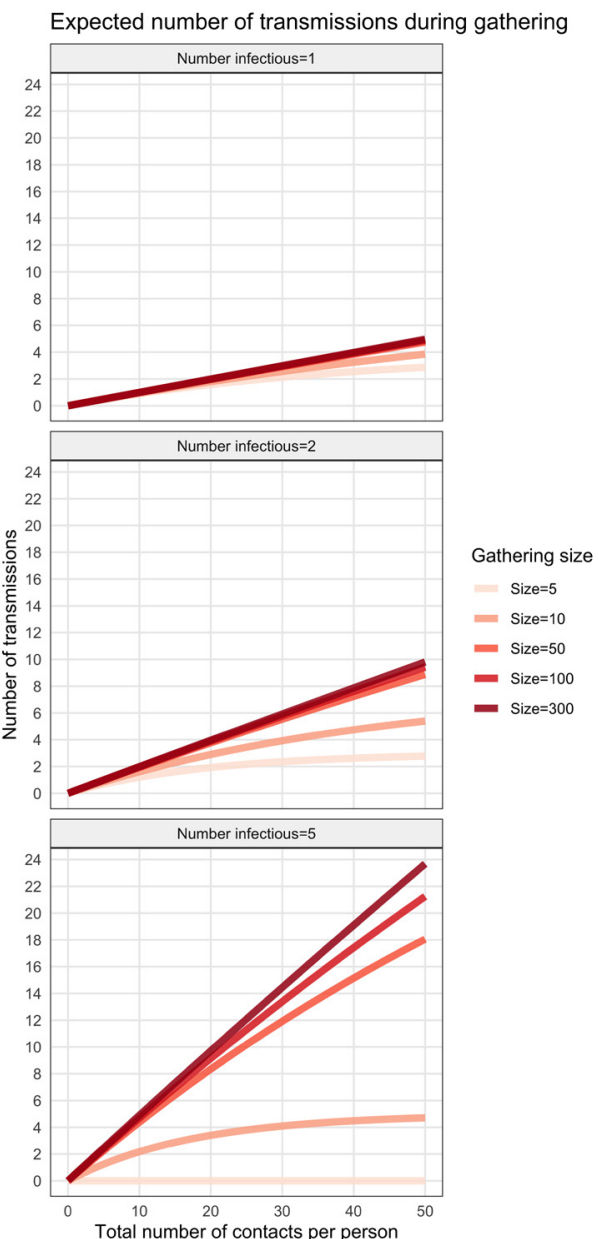

Remarque : Ces graphiques ont été générés en utilisant une probabilité de transmission en cas de contact de $p=10 \%$. Chaque panneau représente un nombre différent de personnes contagieuses participant à un rassemblement (de gauche à droite : 1,2 et 5 ) pour différentes tailles de rassemblement

tailles de rassemblement
(Figure en anglais seulement)

toutes les $S$ personnes susceptibles dans le rassemblement (tous les contagieux devraient contacter $C_{\max }$ fois seulement les personnes susceptibles). La remise à l'échelle de $A$ à la taille du rassemblement donne $a=A / N$. Ce rapport a peut servir de valeur seuil pour évaluer si l'événement extrême où toutes les personnes susceptibles pourraient être infectées pendant le rassemblement. Si prév iest la prévalence de la population, avoir prév $\approx a$ signifie qu'il est possible que toutes les personnes susceptibles soient infectées. Plus généralement, si prév $\approx f \times a$, alors une fraction $f$ des participants susceptibles risque d'être infectée pendant le rassemblement. Par exemple, un rassemblement de 1000 personnes où le nombre maximal de contacts pour une personne est de 30 et la probabilité que I'infection soit transmise au cours d'un contact est de $60 \%$ a une valeur seuil de $a=5,5 \%$. Par conséquent, une prévalence de population supérieure à $5,5 \%$ (i.e. si nous attendons plus de 55 participants contagieux) serait inquiétante pour ce rassemblement, car il existe un potentiel $d$ 'infection pour chaque participant susceptible. Si la prévalence de la population était de $2,75 \%$, la moitié des participants susceptibles risqueraient d'être infectés $(f=0,5)$.

La durée du rassemblement influe également sur le risque de transmission. Normalement, plus les personnes sont ensemble longtemps, plus les possibilités de contacts transmettant le virus sont nombreuses. L'effet du temps sur les transmissions peut être modélisé par une analyse de survie. La proportion de personnes susceptibles restant $t$ unités de temps après le début du rassemblement $(t=0)$ est :

$$
S(t)=e^{-\lambda t}
$$

Le risque d'infection $\lambda$ (supposé constant ici) peut être estimé à partir des contagions enregistrées pendant les rassemblements observés (par la recherche des contacts). Cela suppose implicitement que l'instant d'infection est distribué de manière exponentielle. Si $N$ représente la taille du rassemblement, $T$ sa durée et $i$ le nombre total de transmissions qui ont eu lieu au cours de cet événement, une estimation naïve du risque d'infection serait la suivante :

$$
\hat{\lambda}=\frac{1}{T} \log \left(\frac{N}{i}\right)
$$

Les études portant sur la recherche de contacts pendant les rassemblements peuvent fournir les données nécessaires pour effectuer cette estimation pour un rassemblement donné. La figure 3 est un exemple de données épidémiologiques utilisées pour informer le modèle de survie. Soulignons que les données recueillies dans le cadre de ces études sont probablement inférieures à la réalité; les rassemblements qui ont attiré l'attention des agents de santé publique en raison du grand nombre de cas secondaires ont probablement plus tendance à être signalés que ceux où il $y$ a eu peu ou pas de transmission. La figure 3 montre également une calibration naïve du risque $d$ 'infection pendant les événements $(\hat{\lambda})$ taux données du tableau $\mathrm{S} 1$ de l'annexe. Les estimations du risque d'infection $\hat{\lambda}$ peuvent aider à éclairer les décisions à prendre sur la restriction de la durée des rassemblements.

\section{Réunions récurrentes}

La deuxième catégorie de rassemblements est celle qui se produit régulièrement avec les mêmes participants. Les employés d'une entreprise, les étudiants et le personnel enseignant d'une école, le personnel hospitalier sont des exemples de participants à ces rassemblements.

\section{Définitions et hypothèses}

Les participants aux rassemblements récurrents forment souvent des cohortes (e.g. des classes d'école, des employés de bureau) au sein desquelles les personnes interagissent de manière préférentielle. Le regroupement en cohorte a également été considéré comme une mesure d'atténuation de la transmission pendant les rassemblements (4). En outre, une intervention courante de la santé publique pour minimiser la transmission 
Figure 3 : Risque d'infection estimé à partir de données épidémiologiques provenant de rassemblements sociaux

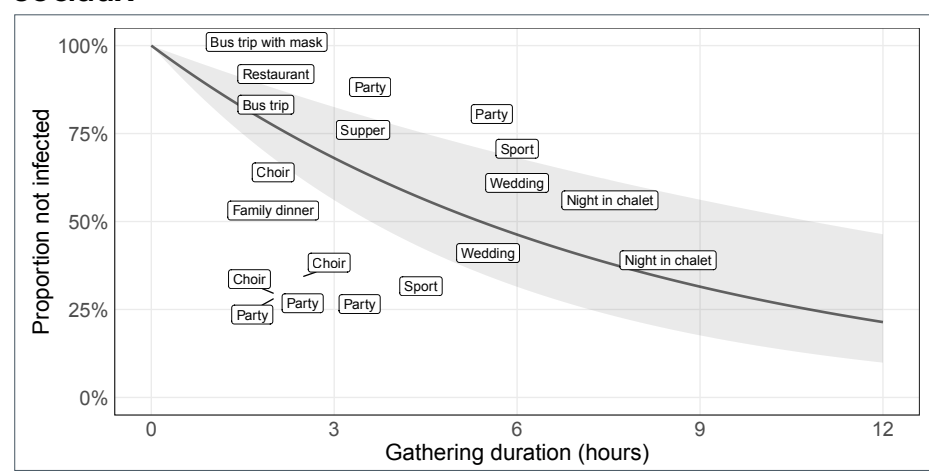

Remarque : Exemple d'ajustement naïf aux données épidémiologiques présentées au Tableau S1 de l'annexe. Chaque étiquette représente le type de rassemblement; sa position sur le graphique indique sa durée approximative (axe horizontal) et la proportion de participants qui n'étaient pas infectés (axe vertical). La courbe noire pleine est la régression linéaire effectuée sur l'échelle logarithmique (voir l'annexe pour plus de détails) et le ruban gris représente I'IC à $95 \%$ (Figure en anglais seulement)

pendant des rassemblements consiste à réduire autant que possible le taux de contact entre les cohortes (5).

Si l'on suppose qu'il y a $M$ cohortes, $G_{1}, G_{2}, \ldots, G_{M}$ et, pour simplifier, que toutes les cohortes comptent le même nombre $N$ de personnes, alors un total de $M \times N$ personnes se réunissent sur une base régulière. D'un point de vue épidémiologique, trois voies de transmission principales sont liées aux rassemblements récurrents : l'introduction de personnes infectées dans une cohorte, la transmission au sein d'une cohorte et la transmission entre cohortes (figure 4).

\section{Risque d'introduction}

Pour les rassemblements récurrents, le risque d'introduction peut être estimé de manière similaire à celui des rassemblements uniques, mais la fréquence à laquelle le rassemblement se produit $(t)$ also needs to be considered. doit également être prise en compte. On estime alors le risque d'introduction dans un rassemblement récurrent dans une collectivité avec prévalence (prév), la taille du rassemblement $(M N)$, constitué de $M$ groupes de taille $N$ pendant $t$ jours.

$$
p_{\text {intro }}=1-(1-\text { prév })^{t M N}
$$

La figure 5 montre que pour un rassemblement récurrent de 100 personnes en cohortes de différentes tailles (20 groupes ou cohortes de 5 personnes, 10 groupes de 10 personnes ou cinq groupes de 20 personnes), la taille de la cohorte ne change pas le risque d'introduction dans le rassemblement dans son ensemble. Cependant, le risque d'introduction dans chaque cohorte baisse considérablement lorsqu'on réduit la taille de la cohorte. Il faut donc élaborer des stratégies pour garantir que toute infection introduite dans l'une des cohortes ne se propagera pas dans les autres cohortes au cours du rassemblement.
Figure 4 : Voies de transmission reliées aux rassemblements récurrents

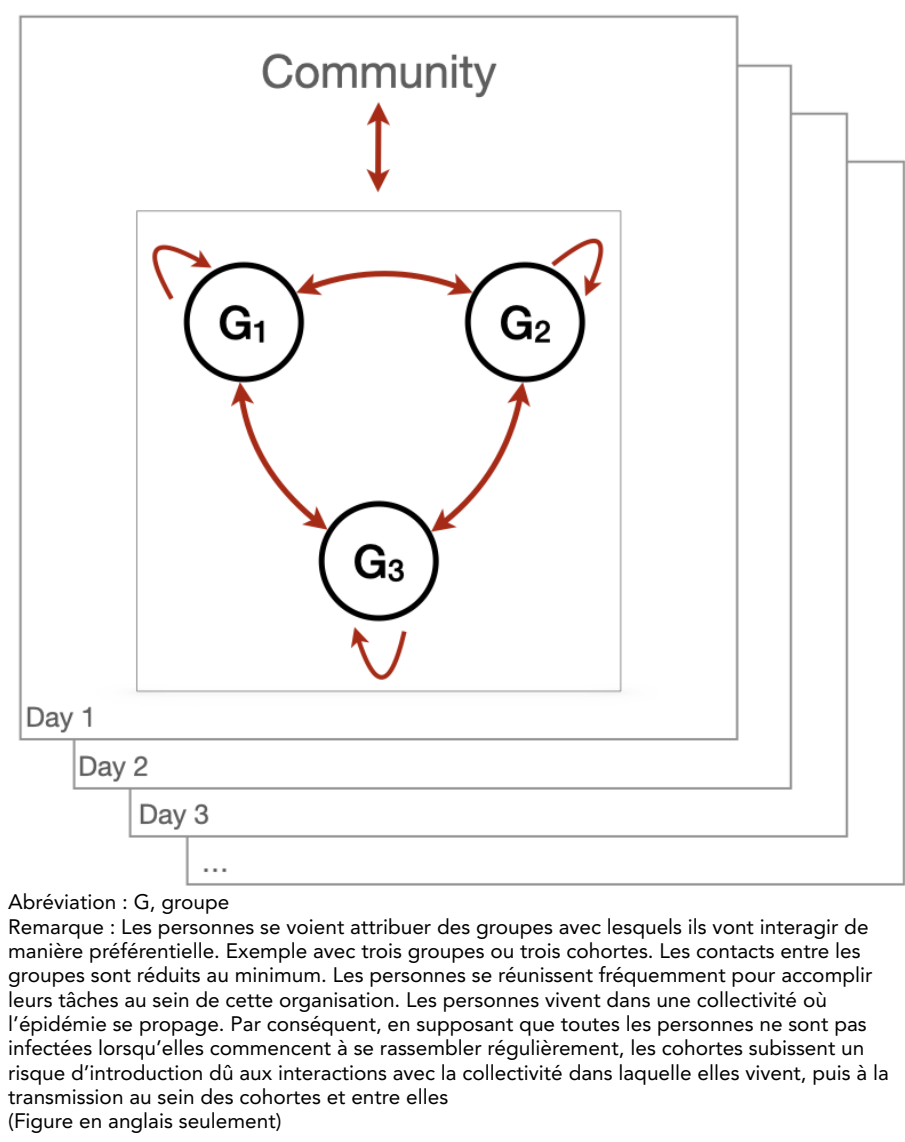

Figure 5 : Risque d'introduction en fonction du temps et de la structure de la cohorte

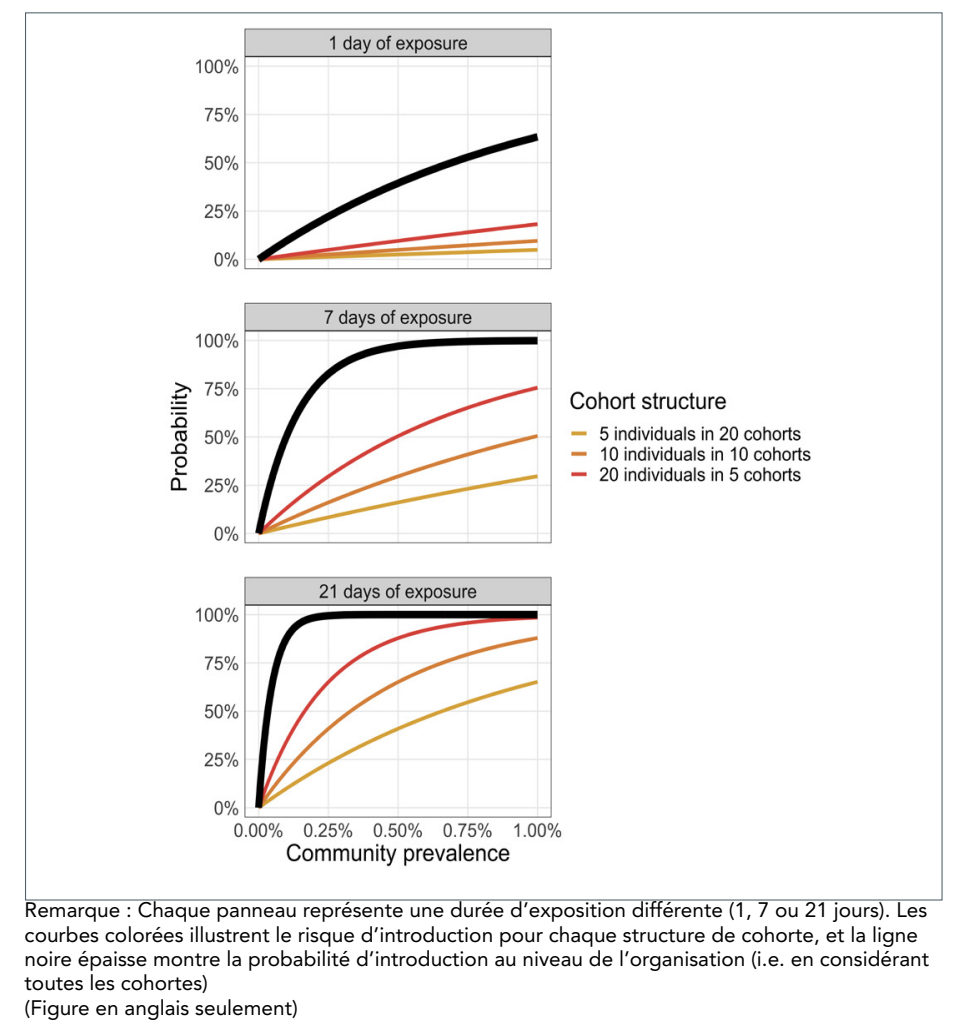


Le risque d'infection provenant de la collectivité est simplement la prévalence de l'infection dans la collectivité (en supposant que le rassemblement est représentatif de la population).

Comme il est expliqué ci dessus dans le cas des rassemblements uniques, si les personnes sont issues d'une sous-population ayant une prévalence prév $_{G^{\prime}}$ différente de celle calculée pour leur collectivité, la prévalence attendue peut être ajustée en utilisant un risque relatif estimé ou un rapport de cotes.

\section{Transmission au sein d'une cohorte}

L'estimation de la transmission au sein d'une cohorte est similaire à l'analyse présentée ci dessus pour les rassemblements uniques, mais avec une valeur plus élevée du nombre de contacts $(C)$ en raison de la nature récurrente du rassemblement.

\section{Transmission entre cohortes}

La probabilité de transmission pendant la durée de l'infectiosité entre une cohorte dont au moins un membre est contagieux et toute autre cohorte totalement susceptible est $p_{\mathrm{bw}}$. Si les cohortes sont complètement isolées, $p_{\mathrm{bw}}=0$, alors le nombre maximum de transmissions secondaires dues à l'introduction d'une personne contagieuse dans une cohorte est limité à la taille de la cohorte, $N$. Rappelons qu'il y a un total de $M \times N$ personnes ( $M$ cohortes avec $N$ personnes chacune), donc le taux d'attaque global ne peut pas dépasser $N / N M=1 / M$. Par exemple, une entreprise qui compte 20 employés répartis en quatre cohortes, chacune comptant cinq personnes, aura un taux d'attaque maximal de $1 / 4=25 \%$ si ces cohortes sont maintenues complètement isolées.

Bien entendu, I'hypothèse d'un isolement complet entre les cohortes est rarement réaliste, et la probabilité de transmission entre les cohortes est supérieure à zéro $\left(p_{\mathrm{bw}}>0\right)$. Si $a$ is est le taux d'attaque au sein d'une seule cohorte $(0 \leq a \leq 1)$, en supposant qu'aucune des contagions n'est détectée, le nombre attendu de personnes infectées dans une cohorte où la personne contagieuse initiale a été introduite est $a N$. Si l'on considère que la cohorte infectée peut potentiellement infecter n'importe quelle autre cohorte en même temps (ce qui revient à ne considérer que deux générations synchrones d'infections ainsi qu'un mélange homogène), le taux d'attaque global est le suivant :

$$
a_{\text {global }}=a\left(\frac{1}{M}+\left(1-\frac{1}{M}\right) p_{\mathrm{bw}}\right)
$$

Lorsque les cohortes sont bien isolées ( $p_{\mathrm{bw}}$ est très petit), le taux d'attaque global est réduit par le simple fait de diviser l'organisation en cohortes $M$ et on a $a_{\text {global }} \approx a / M:$ seule la cohorte qui subit une introduction est affectée, donc le taux d'attaque global est dilué par le nombre de cohortes. À l'autre extrême (figure 6, panneau de droite), si les cohortes sont mal isolées ( $p_{\mathrm{bw}}$ proche de 1), le cloisonnement de l'organisation en cohortes a peu d'effet $\left(a_{\text {global }} \approx a\right)$. Pour des probabilités de transmission entre cohortes faibles à modérées (figure 6 , panneaux de gauche et du centre), l'augmentation du nombre de cohortes dilue nettement le taux d'attaque global ( $a_{\text {global }}$ ) lorsque le taux d'attaque de la cohorte (a) est important (disons supérieur à $20 \%$ ). De plus, en raison des termes $1 / M$, la dilution du taux d'attaque sature lorsque $M$ augmente (figure 6).

\section{Figure 6 : Risque de transmission entre cohortes après} une seule introduction

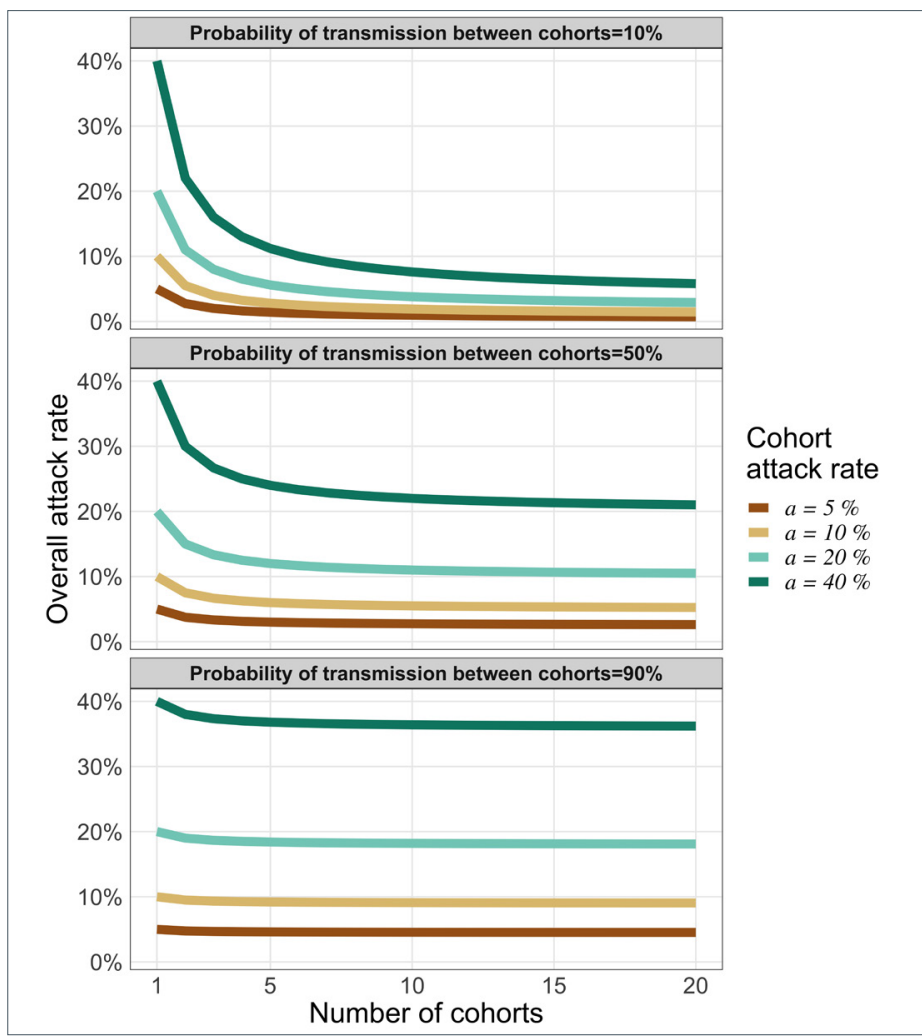

Remarque : L'axe vertical représente le taux d'attaque global pour une organisation qui a séparé ses membres en cohortes (axe horizontal). Chaque courbe colorée représente un taux d'attaque de cohorte différent. Chaque panneau illustre la manière dont le taux d'attaque global (pour l'ensemble de l'organisation) varie en fonction de trois niveaux d'isolement entre les cohortes (isolement élevé pour le panneau de gauche, modéré pour le panneau du centre et faible pour le panneau de droite)

(Figure en anglais seulement)

\section{Atténuation à l'aide de tests}

On peut réduire le risque d'infection pendant un rassemblement en limitant les contacts, en réduisant la probabilité de transmission en cas de contact, ou les deux. La distanciation physique, par exemple en maintenant une distance d'au moins deux mètres entre les participants, peut réduire la probabilité de contact. Il a été démontré que le lavage des mains, I'assainissement des surfaces et l'utilisation correcte des masques réduisent la probabilité de transmission.

Une troisième stratégie pour limiter le risque de transmission consiste à tester les participants avant (pour les rassemblements uniques) ou pendant (pour les rassemblements récurrents) l'événement.

\section{Tests avant le rassemblement}

Il existe actuellement deux types de tests pour diagnostiquer une contagion par le SRAS-CoV-2 : un test basé sur la réaction en chaîne par polymérase (PCR), réalisé dans des laboratoires bien 
équipés, et un test rapide, souvent réalisé au point de service, qui est basé sur l'antigène (e.g. le test rapide PanBio ${ }^{\mathrm{TM}}$ COVID-19 $\mathrm{Ag}$, Abbott Point of Care Inc.). Le premier est considéré comme I'étalon-or, mais il s'accompagne généralement d'un long délai d'exécution, ce qui peut rendre son utilisation peu pratique avant un rassemblement. Le deuxième pourrait être effectué juste avant un rassemblement afin de dépister les participants infectés, mais il n'a qu'une faible sensibilité sur des personnes asymptomatiques (6). L'analyse d'échantillons de salive, qui sont moins invasifs à obtenir que les écouvillons nasopharyngés utilisés actuellement pour les tests PCR, permettrait d'effectuer ce test plusieurs fois (7). L'application routinière de tests pour mieux dépister la transmission dans les rassemblements et dans les lieux de travail est un domaine de recherche en cours (8).

En supposant que l'on surmonte tous les obstacles logistiques liés à la réalisation de tests peu avant un rassemblement, il serait possible de réduire le risque de transmission en testant les participants pendant le rassemblement.

L'estimation du risque de transmission doit tenir compte des différentes durées pendant lesquelles les contagions peuvent être détectées. Dans un scénario où l'excrétion virale dure pendant $D$ jours après le jour de l'infection, la période d'incubation est de $B$ jours, la concentration virale minimale détectable est atteinte après $\delta$ jours, et la fraction asymptomatique de l'infection dans la population est de $\alpha$.

Nous avons supposé qu'une personne infectée ne se rendrait pas à un rassemblement une fois les symptômes apparus. Ainsi, pour les personnes symptomatiques, la fenêtre pour les identifier est de $(B-\delta)$ jours sur une période totale de $B$ jours. En revanche, pour les personnes infectées mais asymptomatiques, la fenêtre pour les identifier est plus longue, $D-\delta$ jours sur un total de $D$ jours (voir la figure 7 ). On a supposé que les personnes symptomatiques ne participeraient à un rassemblement que pendant leur période contagieuse présymptomatique.

Figure 7 : La fenêtre de détectabilité de l'infection virale varie entre les personnes symptomatiques et asymptomatiques

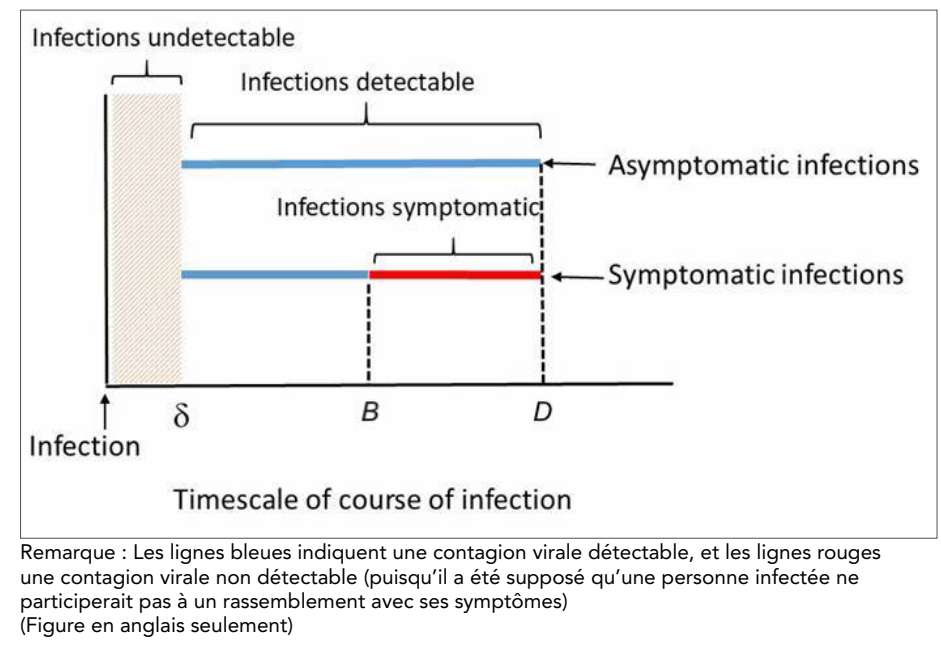

Par conséquent, la probabilité qu'une personne contagieuse soit testée pendant que la charge virale se trouve dans la fenêtre détectable est la suivante :

$$
p_{\text {détectable }}=(1-\alpha) \frac{B-\delta}{B}+\alpha \frac{D-\delta}{D}
$$

Par exemple, en prenant les paramètres typiques d'une contagion par le SRAS-CoV 2, nous avons $B=5$ jours, $D=20$ jours (9), $\alpha=30 \%$ and $\delta=1$ jours; nous avons $p_{\text {détectable }}=84,5 \%$. Autrement dit, environ un participant contagieux sur six ne se trouvera pas dans la fenêtre de détectabilité de l'infection virale.

\section{Atténuation du risque d'introduction et de transmission par des tests}

Il existe de nombreux moyens, dont la plupart dépendent du contexte, pour réduire le risque d'introduction et de transmission dans les rassemblements récurrents. Dans cette section, nous nous concentrons sur l'atténuation du risque de transmission à l'aide de tests périodiques.

Pour réduire le risque d'introduction et de transmission à $d^{\prime}$ autres cohortes (et à la collectivité), nous pouvons tester périodiquement, disons tous les $\tau$ jours, tous les personnes de toutes les cohortes. On suppose que la durée de l'infectiosité est fixée à $D$ jours et qu'il existe un test permettant de détecter I'infection avec une spécificité $s p$ et une sensibilité se. Notez que la détection peut se faire à $n^{\prime}$ importe quel moment de la période d'infectiosité et pas seulement au début (figure 8).

\section{Figure 8 : Tests périodiques relatifs à la période contagieuse}

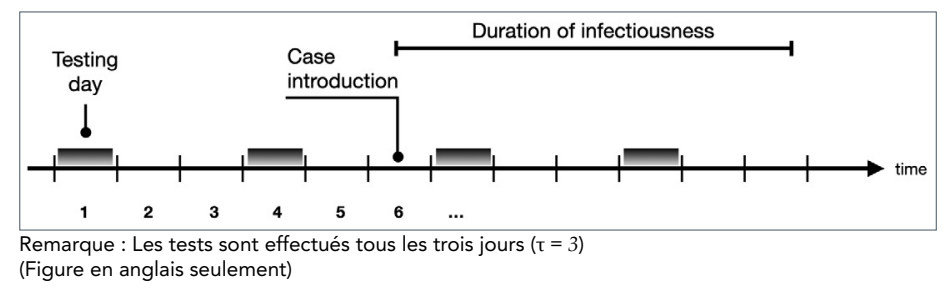

La probabilité d'évaluer l'absence d'une maladie dans un groupe en effectuant plusieurs séries de tests a été largement abordée en épidémiologie vétérinaire et est souvent appelée « absence de maladie » (10). Compte tenu d'une sensibilité se pour un test effectué sur $n$ personnes tous les $t$ jour sur $T$ jours, la probabilité de détecter une contagion est :

$$
p_{\text {détection }}=1-(1-p r e ́ v \times s e)^{n T / \tau}
$$

où prév est la prévalence dans le groupe testé (11). Notez que $p_{\text {détection }}$ peut surestimer la probabilité réelle si les tests périodiques sont corrélés entre eux (e.g. lorsqu'on teste les mêmes personnes).

Pour maximiser la probabilité de détection, les tests pourraient être effectués quotidiennement. Cela est de plus en plus possible grâce aux tests antigéniques réalisés au point de service. Cependant, si le test a une spécificité sous-optimale, 
les faux positifs pourraient imposer des contraintes inutiles (comme la fermeture du lieu de rassemblement ou l'isolement du personnel) à l'organisation (école, entreprise, hôpital). La probabilité que, lors du test de $n$ personnes non infectées, au moins un test renvoie un résultat faussement positif pendant cette période est (voir l'annexe pour plus de détails).

$$
p_{\text {fausse alarme }}=1-s p^{n T / \tau}
$$

La figure 9 illustre l'équilibre entre la maximisation de la probabilité de détection ( $p_{\text {détection }}$ ) et la minimisation de la nuisance des fausses alarmes $\left(p_{\text {fausse alarme }}\right)$ lors du choix de la fréquence des tests $(\tau)$ et de la taille de l'échantillon à tester au sein des groupes $(n)$.

\section{Figure 9 : Compromis entre la probabilité de détecter un cas contagieux et un faux positif}

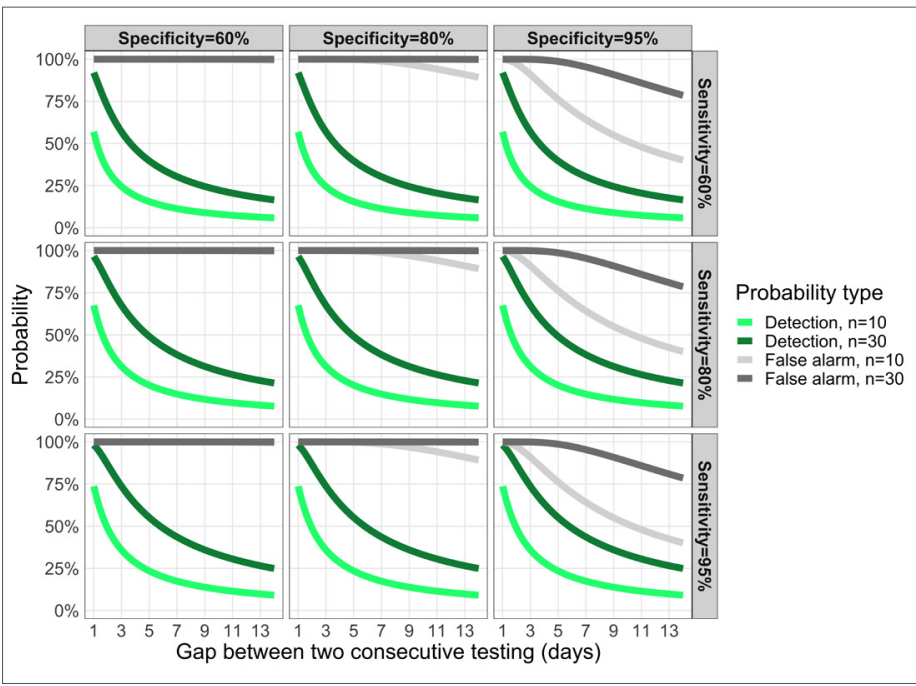

Remarque : Compromis entre la probabilité de détecter un cas contagieux et la probabilité d'un faux positif en fonction de la fréquence des tests (axe horizontal; 1 signifie un test quotidien, 7 signifie un test hebdomadaire). Les courbes vertes représentent la probabilité de détecter la première personne pendant sa période contagieuse, ici fixée à $D=14$ jours lorsque l'on teste n personnes dans l'organisation. Les courbes grises représentent la probabilité d'avoir un faux positif pour $\mathrm{n}$ personnes testées. Chaque panneau présente des valeurs différentes de spécificité et de sensibilite du test (le panneau superieur gauche est le moins précis, le panneau inférieur droit est le plus précis)

(Figure en anglais seulement)

\section{Délai entre l'infection et sa détection}

Compte tenu de la fréquence et de la précision des tests, quelle est la durée prévue entre l'introduction d'un cas contagieux et sa détection? Si nous supposons qu'une personne peut être infectée à tout moment entre deux tests consécutifs, nous pouvons montrer que le temps entre l'infection et sa découverte est limité de la façon suivante:

$$
t_{\text {découverte }} \leq \min \left(D, \tau\left(\frac{1}{s e}-\frac{1}{2}\right)\right)
$$

L'effet de la sensibilité et de la fréquence des tests sur le temps de détection ( $t_{\text {découverte }}$ ) est illustré à la figure 10. Pour une fréquence de test élevée (e.g. moins de tous les trois jours), nous constatons que la sensibilité du test n'a pas beaucoup d'effet sur la rapidité de détection (communication personnelle, Dr Troy Day, Université Queen's, Kingston, ON) (12).
Figure 10 : La fréquence des tests détermine le délai entre l'introduction d'une contagion et sa détection

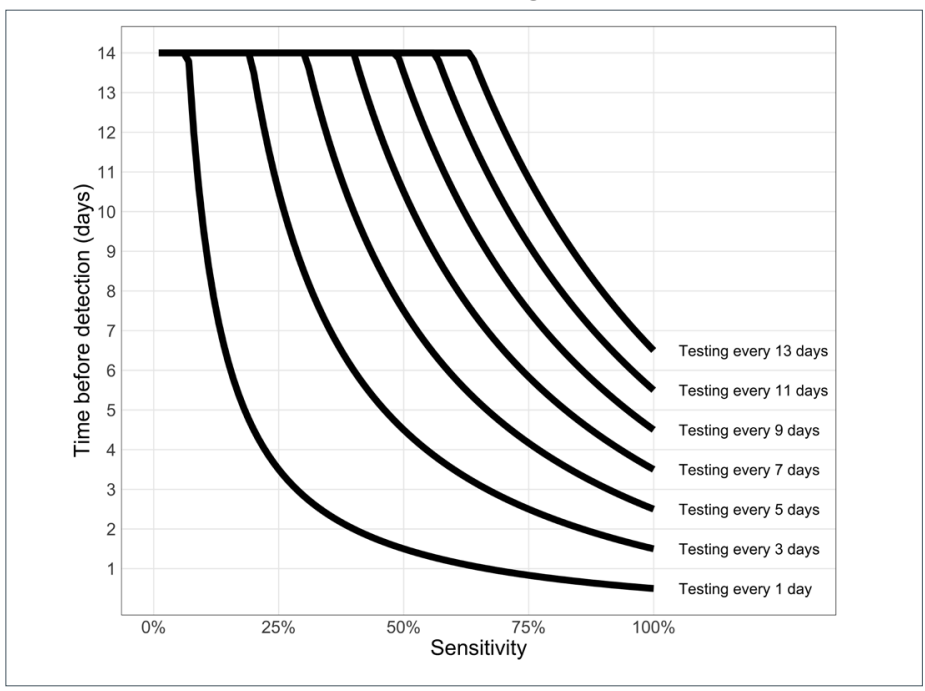

(Figure en anglais seulement)

Une unité de comparaison naturelle pour $t_{\text {découverte }}$ est l'intervalle de génération. L'intervalle de génération est l'intervalle entre le moment où une personne est infectée par une personne contagieuse et le moment où cette personne contagieuse a été infectée. Pour ralentir une épidémie, $t_{\text {découverte }}$ doit être beaucoup plus petit que l'intervalle de génération afin d'éviter les possibilités de transmissions secondaires.

\section{Discussion}

Dans cette étude, nous avons développé un cadre de modèle simpliste et générique pour évaluer le risque de transmission du SRAS-CoV 2 pendant des rassemblements. Ce faisant, nous avons mis en évidence certaines caractéristiques clés des risques que posent les rassemblements ainsi que deux méthodes pouvant atténuer ces risques.

Le premier déterminant du risque pendant les rassemblements est la probabilité qu'au moins une personne contagieuse soit présente ("risque d'introduction »). Ce risque peut être évalué de manière générale en fonction de la prévalence de la population et de la taille du rassemblement. Les propagations massives se produisent souvent pendant des rassemblements (1-3). Intuitivement, limiter la taille des rassemblements réduit la probabilité de ces propagations. Plusieurs études de modélisation ont démontré une association entre la taille de petits rassemblements et de faibles valeurs pour le nombre de reproductions $(13,14)$.

Le deuxième déterminant est le risque de transmission de l'épidémie lors du rassemblement, qui dépend principalement de la taille du rassemblement et du nombre de contacts pendant ce rassemblement. Notre cadre de modélisation simple a mis 
en évidence l'effet de saturation du taux de contact (figure 2). Autrement dit, le risque de transmission n'est nettement réduit que lorsque le taux de contact est suffisamment faible.

Pour les rassemblements récurrents, établir des cohortes réduit généralement le risque de transmission, et les rassemblements comportant un petit nombre de cohortes bien isolées sont moins risqués que ceux comportant un grand nombre de cohortes mal isolées. La façon dont les cohortes sont structurées (peu de cohortes avec beaucoup de personnes, par rapport à beaucoup de cohortes avec peu de personnes) n'a pas d'impact significatif sur la transmission entre les cohortes. Une cohorte plus petite réduira cependant le nombre maximal de personnes pouvant être infectées si une contagion est introduite dans le rassemblement et que les cohortes sont bien isolées.

La probabilité qu'une personne contagieuse se présente au rassemblement est fonction de la prévalence de la COVID-19 au sein de la collectivité. Le test est une option d'atténuation qui pourrait être employée dès l'arrivée des participants au rassemblement; cependant, nous avons démontré que décider de la fréquence des tests avec un test imparfait peut être une question d'équilibre entre l'efficacité de la détection et le désagrément des faux positifs.

Les résultats présentés ici sont généralement en accord avec des modèles plus complexes (3) ainsi qu'avec des approches simples similaires (15). Les limites de cette approche simple pour quantifier le « risque de rassemblement "sont illustrées dans la figure 3, où de nombreux facteurs (e.g. rassemblement à l'intérieur ou à l'extérieur, âge des participants) peuvent influer sur le risque de transmission dans certains rassemblements. À mesure que les connaissances augmenteront grâce aux enquêtes épidémiologiques et aux études prospectives, des valeurs plus précises pour des variables comme les probabilités de transmission permettront d'améliorer la paramétrisation du modèle. Toutefois, l'approche de haut niveau présentée ici ne peut pas remplacer une analyse de modélisation plus approfondie et détaillée qui tient compte les multiples facteurs influant sur le risque de transmission, notamment la quantification et la représentation des types de contact entre les groupes d'âge, les effets de la ventilation, des masques ou de la distanciation physique.

Il y a encore beaucoup d'incertitude quant à la contribution quantitative de la myriade de facteurs qui influencent la transmission du SRAS-CoV 2 dans les rassemblements. Au fur et à mesure que les preuves s'accumuleront, nous serons mieux à même de renseigner les variables qui englobent de multiples facteurs sous-jacents; par exemple, la probabilité de transmission présentée ici devrait être informée par les paramètres en intérieur/extérieur, la distance entre les personnes, I'utilisation du masque, etc. L'énumération exhaustive de ces facteurs et l'évaluation de leur importance concernant le risque de transmission du SRAS-CoV-2 pendant les rassemblements devraient faire l'objet d'études futures.

\section{Conclusion}

Le risque d'introduction peut être évalué de manière générale en fonction de la prévalence de la COVID-19 dans la population et de la taille du rassemblement, tandis que le risque de transmission pendant un rassemblement dépend principalement de la taille du rassemblement. Pour les rassemblements récurrents, la structure de la cohorte n'a pas d'effet significatif sur la transmission entre cohortes. Les stratégies de test peuvent atténuer les risques, mais il faudra déterminer la fréquence et la performance des tests pour établir un équilibre entre la détection et les faux positifs.

Le cadre de modélisation simple présenté ici apporte de la clarté dans les interactions entre les variables en jeu (nombre de participants, taux de contact, etc.) dans l'évaluation du risque épidémiologique. Il peut être utilisé pour fournir une première évaluation du risque d'un rassemblement, et de la possibilité d'atténuer le risque. La généralité du cadre de modélisation utilisé ici permet de démêler ces différents facteurs affectant le risque de transmission lors des rassemblements et peut être utile pour la prise de décision en matière de santé publique.

\section{Déclaration des auteurs}

D. C. - Conception, analyse formelle, rédaction de la version originale, rédaction de la révision et de l'édition

A. F. - Conception, analyse de la rédaction, révision de l'écrit, revue critique

N. H. O. - Conception, révision de l'écriture, revue critique

\section{Intérêts concurrents}

Aucun.

\section{Remerciements}

Aucun.

\section{Financement}

Aucun.

\section{Références}

1. Adam DW, Wu P, Wong JY, Lau EH, Tsang TK, Cauchemez S, Leung GM, Lowling BJ. Clustering and superspreading potential of severe acute respiratory syndrome coronavirus 2 (SARS-CoV-2) infections in Hong Kong. Nat Med 2020;26(11):1714-9. DOI PubMed 
2. Kyriakopoulos AM, Papaefthymiou A, Georgilas N, Doulberis M, Kountouras J. The Potential Role of Super Spread Events in SARS-COV-2 Pandemic; a Narrative Review. Arch Acad Emerg Med. 2020;8(1):e74. PubMed

3. Agence de la santé publique du Canada. Note d'information sur la taille des rassemblements et les caractéristiques des événements à risque élevé de transmission; 2020. https:// www.nccmt.ca/fr/covid-19/covid-19-revues-rapides/194

4. Tupper P, Boury H, Yerlanov M, Colijn C. Event-specific interventions to minimize COVID-19 transmission. Proc Natl Acad Sci USA 2020;117(50):32038-45. DOI PubMed

5. Province of Alberta. Stronger public health measures: Gathering restrictions. Government of Alberta; 2020. https:// www.alberta.ca/enhanced-public-health-measures.aspx

6. Corman VM, Haage VC, Bleicker T, Schmidt ML, Muhlemann B, Zuchowski M, Lei WKJ, Tscheak P, Moncke-Buchner E, Mller MA, Krumbholz A, Brexler JF, Drosten C. Comparison of seven commercial SARS-CoV-2 rapid Point-of-Care Antigen tests. medRxiv. 2020.11.12.20230292. (Publié en ligne avant impression). DOI

7. Yee R, Truong T, Pannaraj PS, Eubanks N, Gai E, Jumarang J, Turner L, Peralta A, Lee Y, Bard JF. Saliva is a Promising Alternative Specimen for the Detection of SARS-CoV-2 in Children and Adults. medRxiv. 2020.10.25.20219055. (Publié en ligne avant impression). DOI

8. Zhang K, Shoukat A, Crystal W, Langley JM, Galvani AP, Moghadas SM. Routine saliva testing for the identification of silent COVID-19 infections in healthcare workers. medRxiv. 2020.11.27.20240044. (Publié en ligne avant impression). DOI

9. He X, Lau EH, Wu P, Deng X, Wang J, Hao X, Lau YC, Wong JY, Guan Y, Tan X, Mo X, Chen Y, Liao B, Chen W, Hu F, Zhang Q, Zhong M, Wu Y, Zhao L, Zhang F, Cowling BJ, Li F, Leung GM. Temporal dynamics in viral shedding and transmissibility of COVID-19. Nat Med 2020;26(5):672-5. DOI PubMed
10. Ziller M, Selhorst T, Teuffert J, Kramer M, Schlüter H. Analysis of sampling strategies to substantiate freedom from disease in large areas. Prev Vet Med 2002;52(3-4):333-43. DOI PubMed

11. Cannon RM. Demonstrating disease freedom-combining confidence levels. Prev Vet Med 2002;52(3-4):227-49. DOI PubMed

12. Larremore DB, Wilder B, Lester E, Shehata S, Burke JM, Hay JA, Tambe M, Mina MJ, Parker R. Test sensitivity is secondary to frequency and turnaround time for COVID-19 surveillance. medRxiv. 2020.06.22.20136309. (Publié en ligne avant impression). DOI

13. Saidan MN, Shbool, MA, Arabeyyat OS, Al-Shihabi ST, Al Abdallat Y, Barghash MA, Saidan H. Estimation of the probable outbreak size of novel coronavirus (COVID-19) in social gathering events and industrial activities. Int J Infect Dis 2020;98:321-7. DOI PubMed

14. Scire J, Nadeau S, Vaughan T, Brupbacher G, Fuchs S, Sommer J, Koch KN, Misteli R, Mundorff L, Götz T, Eichenberger T, Quinto C, Savic M, Meienberg A, Burkard T, Mayr M, Meier CA, Widmer A, Kuehl R, Egli A, Hirsch HH, Bassetti S, Nickel CH, Rentsch KS, Kübler W, Bingisser R, Battegay M, Tschudin-Sutter S, Stadler T. Reproductive number of the COVID-19 epidemic in Switzerland with a focus on the Cantons of Basel-Stadt and Basel-Landschaft. Swiss Med Wkly 2020;150:w20271. DOI PubMed

15. Tupper P, Colijn C. COVID-19's unfortunate events in schools: mitigating classroom clusters in the context of variable transmission. medRxiv 2020.10.20.20216267. (Publié en ligne avant impression). DOI 


\section{Annexe}

\section{Probabilité d'introduction dans les rassemblements récurrents}

La probabilité qu'au moins une personne d'un groupe $G_{i}$ soit infectée un jour donné est :

$$
p_{1}=1-(1-\text { prév })^{N}
$$

Pour ce groupe, la probabilité qu'aucune introduction ne se produise pendant $t$ jours consécutifs est $\left(1-p_{1}\right)^{t}$. La probabilité qu'au moins un des groupes $M$ ait une introduction est $1-\left(\left(1-p_{1}\right)^{t}\right)^{M}$, en substituant $p_{1}$ on obtient $p_{\text {intro }}(t)=1-(1-\text { prév })^{t M N}$

\section{Risque de transmission pendant un rassemblement}

En supposant un mélange homogène des participants à un rassemblement, la probabilité qu'une personne susceptible entre en contact avec une personne contagieuse est de :

$$
\mathrm{P}^{\prime} \text { (un susceptible contacte un infectieux) }=\frac{I}{N-1}
$$

Si la personne susceptible a $C$ contacts pendant le rassemblement, la probabilité qu'au moins un de ces contacts soit avec une personne contagieuse est de :

$$
p_{c}=1-\left(1-\frac{I}{N-1}\right)^{C}
$$

\section{Transmission entre cohortes}

Le nombre attendu d'infections secondaires après une seule introduction est de :

$$
\mathrm{E}\left(A^{\text {global }}\right)=a N+a N(M-1) p_{\mathrm{bw}}
$$

Le premier terme $(a N)$ représente le nombre d'infections générées à partir de la cohorte infectée pour la première fois à cause d'une seule introduction. Le second terme représente les contagions ultérieures des cohortes restantes $M-1$. Pour calculer le taux d'attaque global, nous devons normaliser par la taille du groupe, donc diviser par $M N$ donne :

$$
a_{\text {global }}=a\left(\frac{1}{M}+\left(1-\frac{1}{M}\right) p_{\mathrm{bw}}\right)
$$

\section{Probabilité du désagrément}

La probabilité que tous les tests reviennent négatifs chez une personne non infectée testée tous les $\tau$ jours sur $T$ jours est $\mathrm{sp}^{T / \tau}$. De même, si nous considérons maintenant $n$ personnes non infectées, toutes testées tous les $\tau$ jours, la probabilité que tous ces tests reviennent négatifs est $\mathrm{sp}^{T / \tau}$. Par conséquent, la probabilité qu'au moins un test donne un résultat positif (une fausse alerte) pendant cette période est $1-\mathrm{sp}^{n T / \tau}$.

\section{Délai entre la contagion et la détection}

Soit $L_{0}$ la durée entre l'introduction et le test suivant et suppose qu'elle est uniformément distribuée entre 0 et $\tau$. Le nombre de tests faux positifs jusqu'à la détection, $X$, est supposé être distribué géométriquement et nous avons $\mathbb{P}(X=k)=(1-\mathrm{se})^{k}$, où se est la sensibilité du test. La durée théorique de la période avant la détection est alors définie comme suit :

$$
L=L_{0}+\tau X
$$

L'espérance pour $L$ est simplement $\mathbb{E}(L)=\tau(1-$ se)/se où le premier terme provient de l'hypothèse que $L_{0}$ est uniformément distribué et le second terme de la distribution géométrique pour for $X$. La durée de l'infectiosité $D$ étant finie, le temps de détection de l'infection $L$ est naturellement limité par $D$. En

\begin{tabular}{|c|c|c|c|c|c|c|}
\hline Événement & Pays & $\begin{array}{c}\text { Taille du } \\
\text { rassemblement }\end{array}$ & $\mathbf{R}_{l o}$ & $\mathbf{R}_{h i}$ & Durée $(h)^{b}$ & Source \\
\hline Chorale & États-Unis & 61 & 30 & 50 & 2,5 & Tupper et al., 2020 \\
\hline Restaurant & Chine & 83 & 10 & 10 & 2 & Tupper et al., 2020 \\
\hline Fête & Japon & 90 & 5 & 10 & 4 & Tupper et al., 2020 \\
\hline Dîner en famille & Chine & 7 & 3 & 3 & 2 & Tupper et al., 2020 \\
\hline Nuit dans le chalet & France & 10 & 4 & 9 & 8 & Tupper et al., 2020 \\
\hline Nuit dans le chalet & France & 5 & 1 & 3 & 8 & Tupper et al., 2020 \\
\hline Voyage en autobus & Chine & 39 & 5 & 5 & 2 & Tupper et al., 2020 \\
\hline Voyage en autobus avec masque & Chine & 14 & 0 & 0 & 0,83 & Tupper et al., 2020 \\
\hline Souper & Canada & 120 & 24 & s.o. & 3 & CTV news \\
\hline Sport & Canada & 72 & 24 & s.o. & 6 & The National Post \\
\hline Sport & Canada & 21 & 15 & s.o. & 4 & Montreal Gazette \\
\hline Chorale & France & 27 & 19 & s.o. & 2 & Charlotte, 2020 \\
\hline
\end{tabular}
appliquant l'inégalité de Jensen pour la fonction concave $f(x)=$ $\min (x, D)$, nous avons :

$$
\mathbb{E}(\min (L, D)) \leq \min \left(D, \tau\left(\frac{1}{s e}-\frac{1}{2}\right)\right)
$$

\section{Tableau de la source des données}

Tableau S1 : Transmissions observées lors de rassemblements ${ }^{\mathrm{a}}$ 
Tableau S1 : Transmissions observées lors de rassemblements ${ }^{\mathrm{a}}$ (suite)

\begin{tabular}{|c|c|c|c|c|c|c|}
\hline Événement & Pays & $\begin{array}{c}\text { Taille du } \\
\text { rassemblement }\end{array}$ & $\mathbf{R}_{l o}$ & $\mathbf{R}_{h i}$ & Durée $(\mathbf{h})^{\mathrm{b}}$ & Source \\
\hline Mariage & Allemagne & 111 & 61 & s.o. & 6 & Gelderlander \\
\hline Mariage & Australie & 120 & 42 & s.o. & 6 & The Daily Mail \\
\hline Fête & États-Unis & 10 & 7 & s.o. & 3 & Ghinai et al., 2020 \\
\hline Fête & Portugal & 100 & 16 & s.o. & 6 & The Portugal Resident \\
\hline Fête & États-Unis & 25 & 18 & s.o. & 2 & WFAA \\
\hline Fête & États-Unis & 25 & 18 & s.o. & 2 & The Gainesville Sun \\
\hline Chorale & Pays-Bas & 80 & 32 & N/A & 2 & Omroepgelderland \\
\hline
\end{tabular}

Abréviation : s.o., sans objet

astimation basse (haute) des transmissions est donnée par $R_{l o}\left(R_{h i}\right)$

bes durées ont été estimées lorsqu'elles n'étaient pas explicitement disponibles

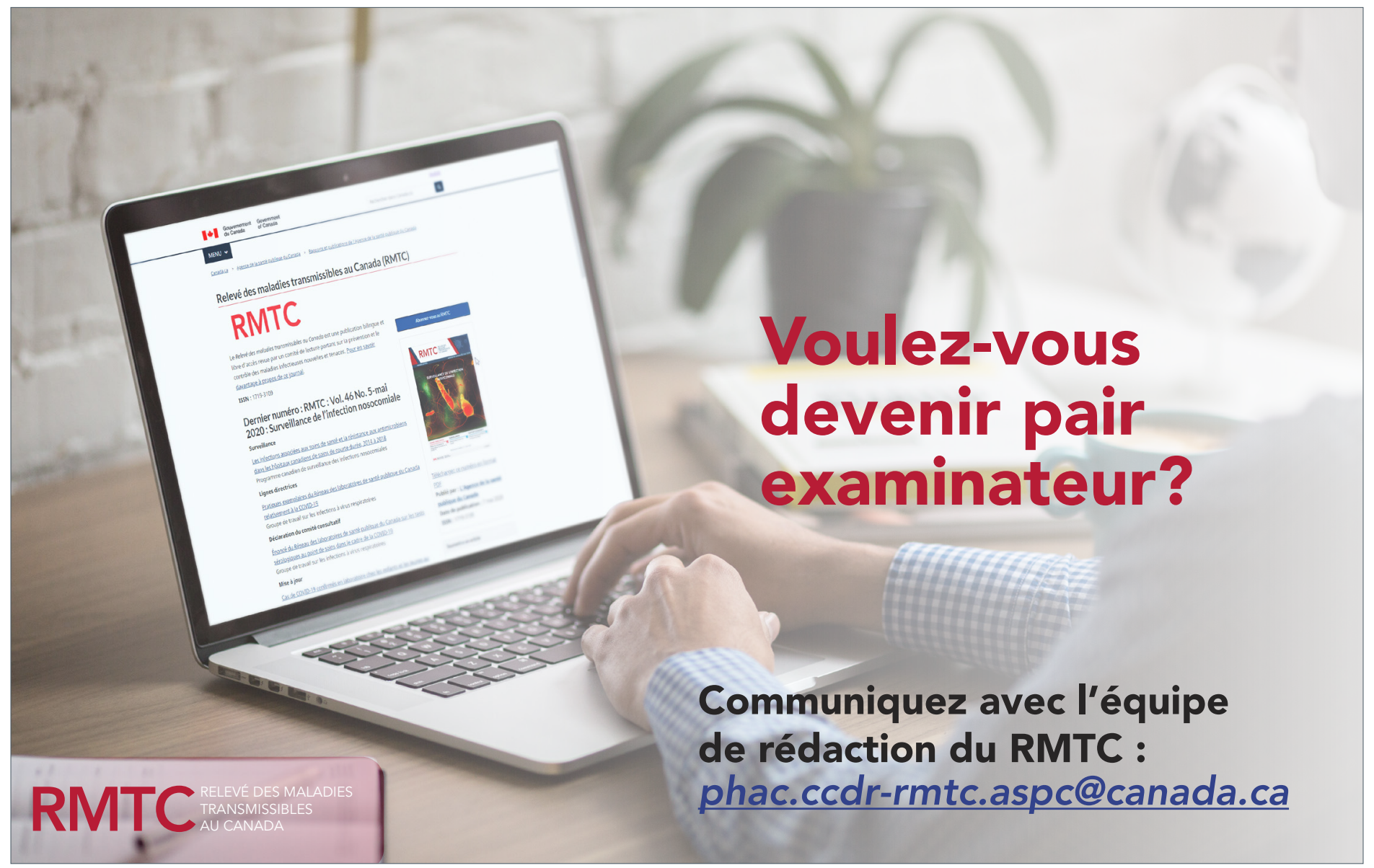

